\title{
Upcoming market catalysts in Q1 of 2010
}

In the first 3 months of 2010, the US FDA is expected to make a decision on marketing applications for several drugs that could advance treatment in their respective indications. Two of these drugs - a once-weekly glucagon-like peptide 1 (GLP1) agonist and an inhalable formulation of insulin - have potential safety concerns that could affect their approvability. Another, already approved drug may have its label expanded to treat hepatic encephalopathy, an area of unmet medical need.

Exenatide LAR (developed by Amylin/ Lilly/Alkermes) is a once-weekly formulation of the approved subcutaneous GLP1 agonist exenatide (Byetta; Amylin/Lilly). Phase III trial results have demonstrated good blood sugar control and weight loss, especially in patients for whom metformin or sulphonylureas are not effective. A once-weekly formulation could enhance convenience and improve patient compliance, depending on the nature of the delivery device. As expected, exenatide LAR has a similar side-effect profile to Byetta. However, exenatide LAR produced thyroid $\mathrm{C}$-cell cancers in rats, an effect that was statistically significant in females at the highest dose tested.

High rates of medullary thyroid cancer that were seen in preclinical studies of another long-acting GLP1 agonist, liraglutide (developed by Novo Nordisk), divided the experts on an advisory committee panel 6-6, with one abstention, in a vote on whether the cancer findings should preclude approval. The FDA is probably using the exenatide LAR preclinical data to help assess whether the observations for liraglutide are a class effect, and its stance on the issue will be clarified by the imminent (at the time of writing) approval decision for liraglutide, which could affect the entire long-acting GLP1 agonist class.

Afresa, a fast-acting insulin inhaler that its developer Mannkind hopes could significantly reduce post-meal glucose fluctuations, faces similar problems. The slightly inferior efficacy of this inhaled formulation compared with injectable insulin should not affect its approvability, especially in type 2 diabetes. However, there are concerns that inhaled insulin could increase the risk of lung cancer, owing to an increased incidence of the disease in clinical trials of the inhaled insulin product, Exubera: 6 out of 4,740 patients treated with Exubera compared with 1 out 4,292 patients treated with comparators. Afresa has reportedly not shown such a discrepancy in its registration programme, but has not yet been as extensively tested as Exubera. The FDA approved Exubera in 2006, but this was before it knew about the potential cancer risk, and so it is unclear how the FDA regards this concern. Even if eventually approved, establishing a market for inhaled insulin could be challenging, as Exubera was withdrawn from the market after 1 year owing to poor sales.

Another drug that is scheduled for review is rifaximin (Xifaxan; Salix Pharmaceuticals), an oral rifamycin-based, gut-selective antibiotic that is minimally absorbed systemically. Originally approved by the FDA in 2004 to treat traveller's diarrhoea, the application for its use in treating hepatic encephalopathy will be analysed by an advisory panel in February, ahead of an FDA approval decision that is anticipated in March. Patients with hepatic encephalopathy, a neuropsychiatric syndrome, are typically treated with diet modifications, lactulose or antibiotics. Lactulose is the most widely used agent but can severely affect the quality of life of the patient. Antibiotics with limited systemic absorption, such as neomycin and metronidazole, can also be used, but some drug absorption can still occur and produce serious toxicities. With a relatively benign adverse-event profile compared with other treatments for hepatic encephalopathy, it seems likely that rifaximin will become a valuable addition to treatment options.

Jesse Rosenthal is a Biotechnology Analyst at Sagient Research Systems, 3655 Nobel Drive Suite 540, San Diego, California 92122, USA. e-mail: jrosenthal@sagientresearch.com 